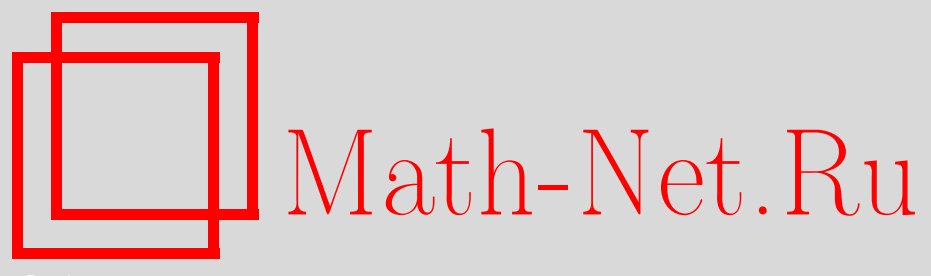

Е. А. Благовещенская, Двойственная структура почти вполне разложимых групп и их колец эндоморфизмов, УМH, 2006, том 61, выпуск 2, 159-160

DOI: https://doi.org/10.4213/rm1733

Использование Общероссийского математического портала Math-Net.Ru подразумевает, что вы прочитали и согласны с пользовательским соглашением http://www . mathnet.ru/rus/agreement

Параметры загрузки:

IP : 54.210 .77 .194

26 апреля 2023 г., $17: 48: 52$

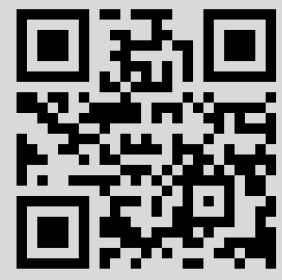




\section{Двойственная структура почти вполне разложимых групп и их колец эндоморфизмов}

\section{Е. А. Благовещенская}

Мы рассматриваем так называемые почти вполне разложимые (пвр-) группы, которые в последние десятилетия приобрели широкую известность, что отражено в монографии А. Мадера [1].

По определению, почти вполне разложимая группа $X$ - это абелева группа без кручения конечного ранга, содержащая некоторую вполне разложимую группу $A$ (прямую сумму групп ранга 1) в качестве подгруппы конечного индекса. Не умаляя общности, считаем, что $A$ - однозначно определенная вполне характеристическая вполне разложимая подгруппа в $X$ конечного индекса, и называем ее регулятором, обозначая $A=R(X)$.

Пусть $\mathscr{A}$ - класс пвр-групп, имеющих регулятор $A$. Известно, что для любой группы $X \in \mathscr{A}$ существует конечное множество простых чисел $P=P(X)$ такое, что $X=\sum_{p \in P} X_{p}$ является суммой своих вполне характеристических подгрупп $X_{p} \in \mathscr{A}$, для которых $X_{p} / A-p$-примарная конечная группа. Обозначим $\mathscr{E}=\operatorname{End} X, \mathscr{E}_{A}=$ End $A, \mathscr{E}_{p}=$ End $X_{p}, \mathscr{H}_{p}=\operatorname{Hom}\left(X_{p}, A\right), e=\exp (X / A)$ и $e_{p}=\exp \left(X_{p} / A\right)$. Для каждого $p \in P$ введем натуральное число $\widehat{e}_{p}=\prod_{q \in P, q \neq p} e_{q}$ и определим группу $X_{p}^{\prime}=\frac{X_{p}}{\widehat{e}_{p}}$ в делимой оболочке группы $X_{p}$, так что $\widehat{e}_{p} X_{p}^{\prime}=X_{p}$. Очевидно, $X_{p} \subset \frac{X_{p}}{\widehat{e}_{p}}$ и $\widehat{e}_{p} \mathscr{E}_{p} \subset \mathscr{E}_{p}$.

Теорема 1. Пусть $X=\sum_{p \in P} X_{p} \in \mathscr{A}$. Канонические иепи из [1; 15.2], $A \subset X \subset$

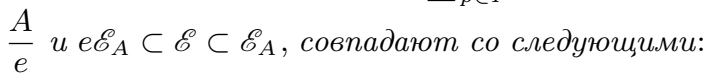

$$
\begin{gathered}
\bigcap_{p \in P} X_{p} \subset \sum_{p \in P} X_{p}=\bigcap_{p \in P} \frac{X_{p}}{\widehat{e}_{p}} \subset \sum_{p \in P} \frac{X_{p}}{\widehat{e}_{p}} u \\
\bigcap_{p \in P} \widehat{e}_{p} \mathscr{E}_{p} \subset \sum_{p \in P} \widehat{e}_{p} \mathscr{E}_{p}=\bigcap_{p \in P} \mathscr{E}_{p} \subset \sum_{p \in P} \mathscr{E}_{p} \text { соответственно. }
\end{gathered}
$$

Кроме того, существует иепь

$$
e \mathscr{E}_{A}=\bigcap_{p \in P} \widehat{e}_{p} \mathscr{H}_{p} \quad \subset \quad \sum_{p \in P} \widehat{e}_{p} \mathscr{H}_{p}=\bigcap_{p \in P} \mathscr{H}_{p} \quad \subset \sum_{p \in P} \mathscr{H}_{p}=\mathscr{E}_{A}
$$

Доказательство основывается на существовании для любого $p \in P$ цепей $A \subset X_{p} \subset$ $\frac{A}{e_{p}}, e_{p} \mathscr{E}_{A} \subset \mathscr{H}_{p} \subset \mathscr{E}_{p} \subset \mathscr{E}_{A}$ и известном факте $\mathscr{E}=\bigcap_{p \in P} \mathscr{E}_{p}$, см. $[1 ; 5.1 .5]$.

Таким образом, $\mathscr{E}$ является как пересечением колец $\mathscr{E}_{p}$, так и суммой двусторонних идеалов $\widehat{e}_{p} \mathscr{E}_{p}$. Данная двойственность позволяет доказать важные факты, касающиеся колец эндоморфизмов $\mathscr{E}$ блочно-жестких пвр-групп $X$, для которых множество критических типов $T_{\mathrm{cr}}(A)$ регулятора $A$, определяемое его разложением $A=\bigoplus_{\tau \in T_{\mathrm{cr}}(A)} A_{\tau}$ на однородные компоненты, состоит из попарно несравнимых типов, см. [2; т. 2, с. 130]. Для таких пвр-групп регулятором является вполне разложимая подгруппа наименьшего индекса. Будет также предполагаться, что все типы $\tau$ из $T_{\mathrm{cr}}(A)$ представляются характеристиками, состоящими только из символов 0 и $\infty$. Почти вполне разложимая группа $X$ с таким регулятором $A$ называется пвр-группой кольцевого типа.

Следующая теорема подготовлена работой [3], в которой показано, что $\mathscr{E}$ и $\mathscr{E} p$, $p \in P$, являются пвр-группами по отношению к операции сложения (что при необходимости обозначается символом $\left.\left({ }^{+}\right)\right)$и справедливо равенство $R\left(\mathscr{E}_{p}^{+}\right)=\operatorname{Hom}\left(X_{p}, A\right)^{+}$, 
кроме того, для кольцевых автоморфизмов верно, что Aut $\mathscr{E}_{p} \subset$ Aut $\mathscr{E}_{A}$, см. [3; теорема 4.5, следствие 3.4].

Теорема 2. Пусть $X=\sum_{p \in P} X_{p} \in \mathscr{A}$ является блочно-жесткой пвр-группой кольцевого типа. Тогда $R\left(\mathscr{E}^{+}\right)=\operatorname{Hom}(X, A)^{+}$и любой автоморфизм кольца $\mathscr{E}$ однозначно продолжается до автоморбизма колъца $\mathscr{E}_{A}$.

ДокАЗАТЕЛЬСтво. Поскольку $\mathscr{H}_{p}^{+} \cong \mathscr{E}_{A}^{+}$является блочно-жесткой вполне разложимой группой, из $[2 ;$ т. $2,86.6$, с. 136$]$ следует, что $\bigcap_{p \in P} \mathscr{H}_{p}$ является вполне разложимой группой как аддитивная структура. Для каждого $p \in P$ существует цепь $\widehat{e}_{p} \mathscr{H}_{p} \subset \widehat{e}_{p} \mathscr{E}_{p} \subset \frac{e \mathscr{E}_{A}}{e_{p}}$, в которой $е \mathscr{E}_{A}=\bigcap_{p \in P} \widehat{e}_{p} \mathscr{E}_{p}$, и $\widehat{e}_{p} \mathscr{H}_{p}$ является вполне разложимой подгруппой наименьшего индекса в $\widehat{e}_{p} \mathscr{E}_{p}$. Из взаимной простоты чисел $\left\{e_{p}: p \in P\right\}$ следует, что аналогичное утверждение верно для подгруппы $\mathscr{H}^{+}=\sum_{p \in P} \widehat{e}_{p} \mathscr{H}_{p}=$ $\bigcap_{p \in P} \mathscr{H}_{p}$ в $\mathscr{E}=\sum_{p \in P} \widehat{e}_{p} \mathscr{E}_{p}$, которая, таким образом, является регулятором (вполне характеристической подгруппой) в $\mathscr{E}^{+}$и, очевидно, совпадает с $\operatorname{Hom}(X, A)^{+}$. Значит, двусторонний идеал $\mathscr{H}=\operatorname{Hom}(X, A)$ кольца $\mathscr{E}$ инвариантен относительно любого $\mathscr{B} \in$ Aut $\mathscr{E}$.

Из $\widehat{e}_{p} \mathscr{E}_{p} \subset \operatorname{Hom}\left(X, X_{p}\right)$ и $e_{p} \operatorname{Hom}\left(X, X_{p}\right) \subset \operatorname{Hom}(X, A)=\mathscr{H}$, снова используя взаимную простоту чисел $\left\{e_{p}: p \in P\right\}$, получаем, что любой автоморфизм кольца $\mathscr{E}=\sum_{p \in P} \widehat{e}_{p} \mathscr{E}_{p}=\bigcap_{p \in P} \mathscr{E}_{p}$ является биекцией на $\widehat{e}_{p} \mathscr{E}_{p}$ и, таким образом, индуцирует автоморфизм на каждом $\mathscr{E}_{p}$, а значит, продолжается до автоморфизма кольца $\mathscr{E}_{A}$. Однозначность следует из $e_{p} \mathscr{E}_{A} \subset \mathscr{E}_{p} \subset \mathscr{E}_{A}$.

Следствие. Aut $\mathscr{E}=\bigcap_{p \in P}$ Aut $\mathscr{E}_{p}$.

\section{Список литературы}

[1] A. Mader, Almost completely decomposable abelian groups, Algebra, Logic Appl., 13, Gordon and Breach, Amsterdam, 2000. [2] Л. Фукс, Бесконечные абелевы группы, т. 1, 2, Мир, М., 1974, 1977. [3] Е. Благовещенская, Фундам. прикл. матем., 10:2 (2004), 23-50.

\section{Е. А. Благовещенская (Е. А. Blagoveshchenskaya)}

Санкт-Петербургский государственный политехнический университет

E-mail: kate@robotek.ru
Представлено А. В. Михалевым Принято редколлегией 01.02 .2006 